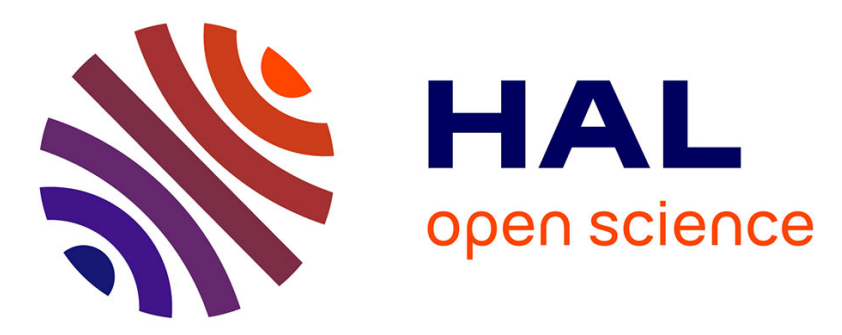

\title{
Formalisme pour le calcul des potentiels adiabatiques d'une molécule diatomique à un électron actif (potentiel - modèle non local). Application aux ions $\mathrm{Na}+2$ et $\mathrm{Ba} 32+$
}

Henri Bergeron, Alexandre Valance

\section{To cite this version:}

Henri Bergeron, Alexandre Valance. Formalisme pour le calcul des potentiels adiabatiques d'une molécule diatomique à un électron actif (potentiel - modèle non local). Application aux ions $\mathrm{Na}+2$ et Ba32+. Journal de Physique, 1989, 50 (20), pp.3109-3122. 10.1051/jphys:0198900500200310900 . jpa-00211129

\section{HAL Id: jpa-00211129 \\ https://hal.science/jpa-00211129}

Submitted on 1 Jan 1989

HAL is a multi-disciplinary open access archive for the deposit and dissemination of scientific research documents, whether they are published or not. The documents may come from teaching and research institutions in France or abroad, or from public or private research centers.
L'archive ouverte pluridisciplinaire HAL, est destinée au dépôt et à la diffusion de documents scientifiques de niveau recherche, publiés ou non, émanant des établissements d'enseignement et de recherche français ou étrangers, des laboratoires publics ou privés. 


\title{
Formalisme pour le calcul des potentiels adiabatiques d'une molécule diatomique à un électron actif (potentiel - modèle non local). Application aux ions $\mathrm{Na}_{2}^{+}$et $\mathrm{Ba}_{2}^{3+}$
}

\author{
H. Bergeron $\left({ }^{1}\right)$ et A. Valance $\left({ }^{2}\right)$ \\ (1) LURE, Bat. 209 D, CNRS-CEA-MEN, Université Paris-Sud, 91405 Orsay Cedex, France \\ (2) S.P.A.S., CEN Saclay, 91191 Gif-sur-Yvette Cedex, France
}

(Reçu le 27 mai 1988, révisé le 22 mai 1989, accepté le 4 juillet 1989)

\begin{abstract}
Résumé. - Nous présentons une méthode de calcul des termes de potentiel adiabatique d'une molécule modélisée par l'interaction d'un électron avec deux cœurs. Le potentiel effectif décrivant l'électron et un cœur est du type potentiel-modèle où interviennent à la fois des états fictifs de cœur et une non-localité. Les problèmes soulevés par ces deux caractéristiques du potentiel sont analysés. Puis cette méthode est testée sur l'ion moléculaire $\mathrm{Na}_{2}^{+}$et enfin appliquée à l'ion $\mathrm{Ba}_{2}^{3+}$.

Abstract. - A calculation method of adiabatic potential energies of a molecule, which is modelised via the interaction of an electron with two cores, is given. The effective potential describing the electron-core interaction is of model potential type which is both characterized with virtual core states and non-local property. The problems corresponding to these two properties of the potential are examined and solved. This method is checked for $\mathrm{Na}_{2}^{+}$molecular ion and applied for $\mathrm{Ba}_{2}^{3+}$ molecular ion.
\end{abstract}

\section{Introduction.}

Les potentiels effectifs atomiques semi-empiriques ont largement été utilisés avec succès pour modéliser un système moléculaire. Dans le domaine de la Physique Atomique et Moléculaire, Bardsley [1], Dixon et Robertson [2] et enfin, Peach [3], présentent des articles de revue sur les différents potentiels semi-empiriques et leurs propriétés.

En particulier, l'approche des potentiels effectifs a été utilisée pour traiter l'interaction d'un électron avec un cœur alcalin [4-6], avec un gaz rare [7, 8] ou avec un ion alcalino-terreux $[9$, 10]. L'ensemble des références données ici n'est pas complet, les articles de revue [1-3] donnent la bibliographie complète sur le sujet.

Dans ces approches, les potentiels utilisés étaient, selon la terminologie de Bardsley, locaux ou non locaux, du type pseudo ou modèle (existence d'états de cœur fictifs) ou mixte [9].

Nous présentons ici une méthode de calcul analytique des termes de potentiels adiabatiques dans le cas d'un potentiel du type Hellmann non local contenant des états fictifs de cœur, 
c'est-à-dire du genre «potentiel-modèle » dépendant du moment angulaire $\ell$ de l'électron. Jusqu'à présent seuls les potentiels-modèles locaux et les pseudo-potentiels locaux on non locaux ont été utilisés dans la littérature.

Le calcul formel des différents éléments de matrice intervenant dans le problème moléculaire de modélisation, dans le cas où le potentiel atomique de départ présente à la fois des états virtuels de cœur et une non-localité (dépendance en $\ell$ : moment angulaire de l'électron), constitue un des apports de cette publication. Les problèmes particuliers soulevés par ces deux caractéristiques sont analysés dans cet article. Les calculs des éléments de matrice propres à ce problème moléculaire, sont traités analytiquement dans le cas d'une base atomique formée de fonctions de type hydrogénoïde. Le problème correspondant au développement sur un centre (par exemple B) du projecteur relatif au centre A ( $\ell$ dépendance)

$$
P_{\mathrm{A}}=\sum_{\ell, m}\left|Y_{\ell, m}\left(\theta_{\mathrm{A}}, \varphi_{\mathrm{A}}\right)\right\rangle\left\langle Y_{\ell, m}\left(\theta_{\mathrm{B}}, \varphi_{\mathrm{B}}\right)\right|
$$

est traité d'une façon à éviter les problèmes de troncature dans la série, par une méthode de moyenne.

Le premier paragraphe de cet article est consacré à la présentation de la méthode de modélisation d'un électron actif en présence de deux cœurs.

Dans le deuxième paragraphe on présente l'aspect calculatoire de l'hamiltonien moléculaire montrant que la méthodologie employée permet de séparer les contributions énergétiques. Dans le paragraphe suivant sont analysés les problèmes dus à l'emploi d'un potentiel non local. Les applications testant, d'une part, la validité de notre approche et donnant, d'autre part, les termes moléculaires de l'ion $\mathrm{Ba}_{2}^{3}{ }^{+}$sont présentés dans le dernier paragraphe.

\section{A. Le modèle moléculaire et les approximations.}

Les unités atomiques sont utilisées dans cet article, sauf avis contraire.

La méthode utilisée ici pour déterminer un potentiel effectif atomique qui permette de reproduire les interactions d'un électron avec un cœur ionique $\mathbf{M}^{z+}$ a été exposée dans les références [10-11]. On donne dans le paragraphe $\mathrm{D}$ les principales caractéristiques de ce potentiel.

L'ion moléculaire $\mathbf{M}_{2}^{(2 z-1)+}$ est considéré comme deux centres $\mathbf{M}^{z+}$ autour desquels gravite un électron. Dans cette représentation chacun des centres est décrit par le potentiel effectif déterminé dans l'étude atomique et la situation propre à ce système est visualisée dans la figure 1.

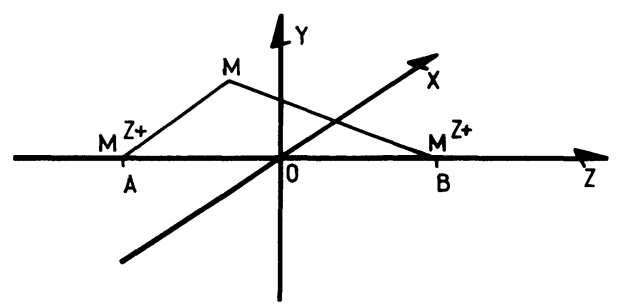

Fig. 1. - Système de référence pour la modélisation des ions homonucléaires à un électron actif en $\mathbf{M}$ et les deux cœurs en $\mathrm{A}$ et $\mathrm{B}$ de charge $Z$. Le milieu de $\mathrm{AB}$ est en $\mathrm{O}$.

[Reference system for one-active-active electron (M) and two identical nuclei (A and $B$ ). The charge is $Z$. $\mathrm{O}$ is the middle of $\mathrm{AB}$.] 
L'hamiltonien $H_{0}$ décrivant cette configuration s'écrit alors :

$$
H_{0}=-\frac{1}{2} \Delta+U_{\mathrm{eff}}^{(\mathrm{A})}+U_{\mathrm{eff}}^{(\mathrm{B})}+V_{\mathrm{pol}}(\mathbf{r}, R)+V_{\mathrm{cc}}(R) .
$$

Les différentes contributions à $H_{0}$ sont définies comme suit :

i) les trois premiers termes décrivent simplement l'électron dans le champ des deux ions ;

ii) le terme $\mathrm{V}_{\text {pol }}(\mathbf{r}, R)$ décrit les effets de polarisation qui sont de trois natures.

Tout d'abord l'électron polarise les ions $\mathbf{M}^{z+}$ au cours de son mouvement. Cette contribution est incluse implicitement dans la valeur des paramètres du potentiel effectif $U_{\text {eff }}$ [12], aussi nous n'ajouterons pas de terme supplémentaire pour représenter cet effet.

Par ailleurs, chaque ion $\mathrm{M}^{z+}$ polarise le cœur de l'autre centre et déforme les orbitales profondes. Dans le cas où notre modèle contient des états de cœur, ceux-ci interviennent dans le calcul moléculaire en donnant naissance à des orbitales virtuelles moléculaires qui peuvent traduire la déformation des orbitales profondes. On peut donc penser qu'on tient compte d'une certaine manière de cet effet de polarisation.

D'autre part, l'existence d'un troisième effet, dit terme « à trois corps » $V_{3}(\mathbf{r}, R)$, qui traduit l'effet couplé de l'électron et de l'ion $\mathbf{M}^{z+}$ sur le cœur de l'autre centre $\mathrm{M}^{z+}$, a une importance qui a été soulignée et calculée maintes fois $[7,8]$. Cette contribution ne peut être reproduite que par l'introduction d'un terme spécifique dans l'hamiltonien. Nous avons négligé ce terme pour ne pas ajouter d'autres paramètres à l'étude moléculaire. Nous savons néanmoins que nous commettons par là-même une erreur systématique sur la valeur des énergies moléculaires. Nos calculs relatifs à l'ion moléculaire $\mathrm{Na}_{2}^{+}$montrent, en les comparant aux résultats bien connus [13-14], que l'on peut évaluer cette erreur à quelques millièmes d'unité atomique. En ce qui concerne l'ion moléculaire $\mathrm{Ba}_{2}^{3+}$ nous prendrons de même :

$$
V_{3}(\mathbf{r}, R)=0
$$

et nous argumenterons pourquoi, à l'heure actuelle, nous ne pouvons améliorer les résultats proposés ;

iii) le terme $V_{\mathrm{cc}}(R)$ est un terme représentant l'énergie de répulsion des deux ions qui est, en première approximation, limité à l'énergie électrostatique, c'est-à-dire :

$$
V_{\mathrm{cc}}(R)=\frac{Z^{2}}{R}
$$

$V_{\mathrm{cc}}(R)$ étant une simple correction scalaire, nous sommes ramenés à diagonaliser $H$ :

$$
H=-\frac{1}{2} \Delta+U_{\mathrm{eff}}^{(\mathrm{A})}+U_{\mathrm{eff}}^{(\mathrm{B})}
$$

les effets de polarisation étant inclus dans $U_{\text {eff }}$ et les orbitales moléculaires de cœur.

Il est clair que dans la description choisie, le cœur de l'ion moléculaire est représenté par la somme des potentiels effectifs des deux centres, et ceci ne peut pas être considéré comme valable dans tous les cas. En effet, s'il est vrai que pour les grandes valeurs de $R$, les deux cœurs n'interfèrent pas, par contre ceci n'est plus vérifié lorsque $R$ à des valeurs proches du rayon de $\mathrm{M}^{z+}$, c'est-à-dire de la portée de la partie de $U_{\text {eff }}$ qui représente le cœur.

Cela signifie que notre modèle sera d'autant mieux adapté à la situation où les distances interatomiques deviennent grandes devant les dimensions de $\mathbf{M}^{z+}$. La base adaptée à la situation asymptotique à l'infini, c'est-à-dire la base atomique, est donc utilisée pour la diagonalisation de $H$. 


\section{B. Calcul de l'hamiltonien moléculaire.}

Dans ce paragraphe nous exposons le calcul de la matrice représentant $H$ dans la base choisie et montrons que deux contributions énergétiques d'origines différentes se séparent.

Introduisons l'opérateur unitaire de translation $\mathcal{F}(\mathbf{a})$ défini par :

$$
\langle\mathbf{r}|\mathcal{F}(\mathbf{a})| \Phi\rangle=\langle\mathbf{r}-\mathbf{a} \mid \Phi\rangle \text {. }
$$

Afin d'alléger l'écriture des formules, utilisons les notations simplifiées suivantes :

$$
\begin{aligned}
t & =\mathcal{F}\left(\frac{\mathbf{R}}{2}\right) \\
T & =t^{2}=\mathcal{F}(\mathbf{R}) .
\end{aligned}
$$

Alors si on note $U_{\text {eff }}$ le potentiel effectif pris dans le repère d'origine $\mathrm{O}$ milieu de $\mathrm{AB}$, l'hamiltonien $H$ s'écrit :

$$
H=-\frac{1}{2} \Delta+t U_{\mathrm{eff}} t^{+}+t^{+} U_{\mathrm{eff}} t
$$

Notons $|\alpha m\rangle(\alpha \equiv n, \ell)$ la base atomique relative à l'origine $\mathrm{O}$ du repère. Les états atomiques du centre A sont alors $t^{+}|\alpha m\rangle$ et ceux du centre B sont $t|\alpha m\rangle$.

L'introduction du projecteur $\left(1+\varepsilon \pi_{z}\right) / 2$ permet d'exprimer la base de $m$ et $\varepsilon= \pm 1$ donnés, soit $\{|\alpha, m, \varepsilon\rangle\}$ par :

$$
|\alpha, m, \varepsilon\rangle=\frac{1+\varepsilon \pi_{z}}{\sqrt{2}} t^{+}|\alpha, m\rangle
$$

l'opérateur $\pi_{z}$ étant associé à la symétrie par rapport au plan Oxy.

Dans le sous-espace de $m$ et $\varepsilon$ donnés, les vecteurs propres de $H$ se présentent sous la forme :

$$
\begin{aligned}
\left|\Phi_{\mathrm{a} m \varepsilon}\right\rangle & =\sum_{\alpha} C_{\alpha \mathrm{a}}^{(m \varepsilon)}|\alpha, m, \varepsilon\rangle \\
H\left|\Phi_{\mathrm{a} m \varepsilon}\right\rangle & =E_{\mathrm{a} m \varepsilon}\left|\Phi_{\mathrm{a} m \varepsilon}\right\rangle
\end{aligned}
$$

où l'index «a » spécifie un état adiabatique moléculaire.

L'élément de matrice de $H$ pour $m$ et $\varepsilon$ donnés entre deux états est donné par:

$$
H_{\beta \alpha}^{(m \varepsilon)}=\langle\beta, m, \varepsilon|H| \alpha, m, \varepsilon\rangle=\frac{1}{2}\left\langle\beta, m\left|t\left(1+\varepsilon \pi_{z}\right) H\left(1+\varepsilon \pi_{z}\right) t^{+}\right| \alpha, m\right\rangle .
$$

$H$ se réduit à :

$$
H_{\beta \alpha}^{(m \varepsilon)}\left\langle\beta, m\left|\left(1+\varepsilon \pi_{z}\right) t H t^{+}\right| \alpha, m\right\rangle
$$

en utilisant la propriété $t \pi_{z}=\pi_{z} t^{+}$.

L'hamiltonien atomique étant noté $h_{\mathrm{a}}$ :

$$
h_{\mathrm{a}}=-\frac{1}{2} \Delta+U_{\mathrm{eff}}
$$

alors :

$$
t H t^{+}=h_{\mathrm{a}}+T U_{\mathrm{eff}} T^{+}
$$


Finalement, après quelques calculs d'algèbre relatifs aux opérateurs, on obtient :

$$
H_{\beta \alpha}^{(m \varepsilon)}=\left\langle\beta, m\left|\left(1+\varepsilon T \pi_{z}\right)\left(h_{a}+T U_{\text {eff }} T^{+}\right)\right| \alpha, m\right\rangle .
$$

On voit apparaître deux contributions à la matrice $H^{(m \varepsilon)}$ par l'intermédiaire de deux opérateurs hermitiques $\mathrm{H}_{1}$ et $\mathrm{H}_{2}$ :

$$
\begin{aligned}
H_{1} & =h_{\mathrm{a}}+T U_{\text {eff }} T^{+} \\
H_{2} & =T \pi_{z}\left(h_{\mathrm{a}}+T U_{\text {eff }} T^{+}\right) \\
H_{\beta \alpha}^{(m \varepsilon)} & =\left\langle\beta, m\left|H_{1}\right| \alpha, m\right\rangle+\varepsilon\left\langle\beta, m\left|H_{2}\right| \alpha, m\right\rangle .
\end{aligned}
$$

En utilisant les propriétés de $T$ et $\pi_{z}$, il est facile de vérifier que l'on peut écrire $\mathrm{H}_{2}$ sous la forme :

$$
H_{2}=\left[T h_{\mathrm{a}}+U_{\mathrm{eff}} T\right] \pi_{z} .
$$

On peut donc isoler les deux contributions physiques au phénomène, sous la forme de deux hamiltoniens $H_{1}$ et $H_{2}$ :

$-H_{1}$ représente un ion $\mathrm{M}^{(z-1)+}$ polarisé par $\mathrm{M}^{2+}$.

- $\mathrm{H}_{2}$ représente l'énergie de mise en commun de l'électron entre les deux centres.

Par ailleurs, les expressions des éléments de matrice de $H_{1}$ et $H_{2}$ peuvent encore être simplifiées en tenant compte du fait que $|\alpha, m\rangle$ est la base qui diagonalise $h_{\mathrm{a}}$, alors :

$$
\begin{gathered}
\left\langle\beta, m\left|H_{1}\right| \alpha, m\right\rangle=E_{\alpha}^{(\text {a) }} \delta_{\beta \alpha}+\left\langle\beta, m\left|T U_{\text {eff }} T^{+}\right| \alpha, m\right\rangle \\
\left\langle\beta, m\left|H_{2}\right| \alpha, m\right\rangle=(-1)^{\ell_{\alpha}+m}\left\{E_{\alpha}^{(a)}\langle\beta, m|T| \alpha, m\rangle+\left\langle\beta, m\left|U_{\text {eff }} T\right| \alpha, m\right\rangle\right\} .
\end{gathered}
$$

On remarque alors que seulement trois types d'éléments de matrice sont à calculer.

\section{Les problèmes propres au potentiel non local.}

Le problème que pose l'emploi de $U_{\text {eff }}$ réside dans la troncature de la série en $\ell$. En effet, nous ne connaissons pas $U_{\text {eff }}$ comme une série infinie, mais seulement comme une somme sur $\ell=0,1,2,3$ (voir Tabs. Ia, Ib et III).

Un calcul simple montre que la connaissance partielle de $U_{\text {eff }}$ n'entraîne aucune approximation pour le calcul de $\left\langle\beta, m\left|U_{\text {eff }}\right| \alpha, m\right\rangle$, il est par ailleurs facile de remarquer que le produit scalaire par $\langle\beta, m|$ réduit la contribution de $U_{\text {eff }}$ au seul terme en $\ell_{\beta}$.

Par contre, ceci n'est plus vrai pour $\left\langle\beta, m\left|T U_{\text {eff }} T^{+}\right| \alpha, m\right\rangle$. En effet, cet élément de matrice fait intervenir les fonctions translatées $T^{+}|\alpha, m\rangle$ et $T|\beta, m\rangle$. Or si les états atomiques $|\alpha, m\rangle$ possèdent une valeur de $\ell_{\alpha}$ définie, les états translatés se décomposent sur toutes les valeurs de $\ell$.

La connaissance incomplète de $U_{\text {eff }}$ va donc entrâner une approximation dans le calcul et ceci sera particulièrement gênant pour les états $\ell_{\alpha}=2,3$ car $U_{\text {eff }}$ ne contient pas de terme en $\ell$ de valeur supérieure.

De ces différentes constatations, on peut conclure que nous n'aurons pas de difficulté à calculer les termes $\left\langle\beta, m\left|H_{2}\right| \alpha, m\right\rangle$ relatifs à la mise en commun de la charge. Par contre, le problème se posera pour $\left\langle\beta, m\left|H_{1}\right| \alpha, m\right\rangle$ décrivant la polarisation de $\mathrm{M}^{(z-1)+}$ par $\mathrm{M}^{2+}$.

Pour pallier à cette difficulté, examinons l'expression explicite de $U_{\text {eff }}$ que nous utilisons :

$$
U_{\text {eff }}=-\frac{z}{r}-\sum_{\ell} \frac{Z_{\ell}-z}{r} \mathrm{e}^{-\sigma_{\ell} r} \sum_{m}|\ell m\rangle\langle\ell m| .
$$


Tableau Ia. - Valeur des coefficients $\left(Z_{\ell}\right.$ et $\left.\sigma_{\ell}\right)$ du pseudo-potentiel de Hellmann: $-\Sigma_{\ell} \frac{Z_{\ell}-z}{r} \mathrm{e}^{-\sigma_{\ell} r} \sum_{m}\left|\ell_{m}\right\rangle\langle\ell m|$ décrivant l'atome de sodium. Les valeurs expérimentales $E^{\exp }$ des énergies d'excitation sont comparées aux valeurs calculées $E^{\mathrm{c}}$ par la donnée des pourcentages d'erreur en dernière colonne du tableau. La correspondance entre états expérimentaux et pseudo-états est indiquée dans les colonnes 1 et 3.

$\left[Z_{\ell}\right.$ and $\sigma_{\ell}$ values corresponding to the Hellmann pseudo-potential : $-\sum_{\ell} \frac{Z_{\ell}-z}{r} \exp \left(-\sigma_{\ell} r\right) \sum_{m}|\ell m\rangle\langle\ell m|$. The values correspond to $\mathrm{Na}$ atom. In the last column are reported comparisons (error percentage) between the $E^{\exp }$ experimental values and our calculated values. The correspondance between experimental states and pseudo-states is reported columns 1 and 3.]

\begin{tabular}{|c|c|c|c|c|c|}
\hline \multirow{2}{*}{$\begin{array}{l}C_{0}=-1,685 \\
\sigma_{0}=1,446\end{array}$} & \multirow[t]{2}{*}{$\mathrm{Na}$} & \multirow[t]{2}{*}{$E^{\exp }$ (u. a. ) } & \multirow[t]{2}{*}{$h$} & \multirow[t]{2}{*}{$E^{\mathrm{c}}$ (u. a. ) } & \multirow[t]{2}{*}{$\%$ erreur } \\
\hline & & & & & \\
\hline & $3 \mathrm{~s}$ & $-0,18886$ & $1 \mathrm{~s}$ & $-0,18886$ & 0,0 \\
\hline & $4 s$ & $-0,071579$ & $2 s$ & $-0,068330$ & 4,5 \\
\hline & $5 s$ & $-0,037585$ & $3 s$ & $-0,03463$ & 7,0 \\
\hline & $6 s$ & $-0,023132$ & $4 s$ & $-0,02084$ & 9,0 \\
\hline & $7 \mathrm{~s}$ & $-0,0156627$ & $5 s$ & $-0,013993$ & 10,0 \\
\hline \multicolumn{6}{|l|}{$\begin{array}{l}Z_{1}=-1,685 \\
\sigma_{1}=1,446\end{array}$} \\
\hline & $3 p$ & $-0,11155$ & $2 p$ & $-0,11155$ & 0,0 \\
\hline & $4 p$ & $-0,050935$ & $3 p$ & $-0,051086$ & 0,3 \\
\hline & $5 p$ & $-0,029195$ & $4 p$ & $-0,029223$ & 0,1 \\
\hline & $6 p$ & $-0,018919$ & $5 p$ & $-0,018906$ & 0,5 \\
\hline & $7 p$ & $-0,013253$ & $6 \mathrm{p}$ & $-0,013228$ & 0,2 \\
\hline \multicolumn{6}{|l|}{$Z_{2}=-1,685$} \\
\hline \multicolumn{6}{|l|}{$\sigma_{2}=1,446$} \\
\hline & $3 d$ & $-0,55937$ & $3 d$ & $-0,055271$ & 0,0 \\
\hline & $4 d$ & $-0,031442$ & $4 d$ & $-0,031092$ & 1,1 \\
\hline & $5 d$ & $-0,020106$ & $5 d$ & $-0,019910$ & 0,9 \\
\hline & $6 d$ & $-0,013953$ & $6 d$ & $-0,013834$ & 0,8 \\
\hline & $7 d$ & $-0,010246$ & $7 d$ & $-0,010168$ & 0,7 \\
\hline \multirow{2}{*}{\multicolumn{6}{|c|}{$\begin{array}{l}Z_{3}=-1,685 \\
\sigma_{3}=1,446\end{array}$}} \\
\hline & & & & & \\
\hline & $4 \mathrm{f}$ & $-0,312612$ & $4 f$ & $-0,312468$ & 0,0 \\
\hline & $5 \mathrm{f}$ & $-0,020011$ & $5 f$ & $-0,019997$ & 0,0 \\
\hline & $6 f$ & $-0,013895$ & $6 f$ & $-0,013887$ & 0,0 \\
\hline & $7 f$ & $-0,010208$ & $7 f$ & $-0,010203$ & 0,0 \\
\hline & $8 f$ & $-0,0078171$ & $8 f$ & $-0,0078116$ & 0,0 \\
\hline
\end{tabular}

Nous verrons dans l'étude atomique présentée dans les paragraphes suivants, que les paramètres $\sigma_{\ell}$ et $Z_{\ell}$ dépendent peu de $\ell$ dans le cas alcalin (sodium) et dans le cas alcalinoterreux (baryum). L'idée naturelle qui en résulte est de remplacer les termes dépendant de $\ell$ 
Tableau Ib. - Mêmes commentaires que le tableau Ia pour le potentiel-modèle du Na.

[This table corresponds to the model-potential case of $\mathrm{Na}$ atom.]

\begin{tabular}{|c|c|c|c|c|c|c|}
\hline$Z=2203$ & $\mathrm{Na}$ & $E^{\exp }$ (u. a.) & \multirow{7}{*}{ Cœur } & $h$ & $E^{\mathrm{c}}$ (u. a. ) & $\%$ erreur \\
\hline$Z_{0}=2,203$ & & & & $1 \mathrm{~s}$ & $-1,4828$ & - \\
\hline \multirow[t]{5}{*}{$\sigma_{0}=1,1$} & $3 \mathrm{~s}$ & $-0,18886$ & & $2 s$ & $-0,18886$ & 0,0 \\
\hline & $4 s$ & $-0,071579$ & & $3 s$ & $-0,071624$ & 0,06 \\
\hline & $5 s$ & $-0,037585$ & & $4 s$ & $-0,037430$ & 0,4 \\
\hline & $6 s$ & $-0,023132$ & & $5 \mathrm{~s}$ & $-0,022969$ & 0,7 \\
\hline & $7 \mathrm{~s}$ & $-0,0156627$ & & $6 s$ & $-0,015528$ & 0,8 \\
\hline \multicolumn{7}{|l|}{$Z_{1}=6,423$} \\
\hline \multirow[t]{6}{*}{$\sigma_{1}=1,1$} & & & Cœur & $2 \mathrm{p}$ & $-1,1932$ & \\
\hline & $3 p$ & $-0,11155$ & & $3 p$ & $-0,11155$ & 0,0 \\
\hline & $4 \mathrm{p}$ & $-0,050935$ & & $4 p$ & $-0,051443$ & 0,9 \\
\hline & $5 p$ & $-0,029195$ & & $5 p$ & $-0,028842$ & 1,2 \\
\hline & $6 \mathrm{p}$ & $-0,018919$ & & $6 \mathrm{p}$ & $-0,018501$ & 2,1 \\
\hline & $7 p$ & $-0,013253$ & & $7 p$ & $-0,012895$ & 2,5 \\
\hline \multicolumn{7}{|l|}{$Z_{2}=2,1144$} \\
\hline \multicolumn{7}{|l|}{$\sigma_{2}=1,1$} \\
\hline & $\begin{array}{l}3 \mathrm{~d} \\
4 \mathrm{~d}\end{array}$ & $\begin{array}{l}-0,055937 \\
-0,031442\end{array}$ & & $\begin{array}{l}3 d \\
4 d\end{array}$ & $\begin{array}{r}-0,055937 \\
-0,031453\end{array}$ & $\begin{array}{l}0,0 \\
0,03\end{array}$ \\
\hline & $5 d$ & $-0,020106$ & & $5 \mathrm{~d}$ & $-0,020112$ & 0,0 \\
\hline & $6 d$ & $-0,013953$ & & $6 d$ & $-0,013956$ & 0,0 \\
\hline & $7 d$ & $-0,010246$ & & $7 d$ & $-0,010247$ & 0,0 \\
\hline \multirow{2}{*}{\multicolumn{7}{|c|}{$\begin{array}{l}Z_{3}=2,962 \\
\sigma_{3}=1,1\end{array}$}} \\
\hline & & & & & & \\
\hline & $4 f$ & $-0,312612$ & & $4 f$ & $-0,312612$ & 0,0 \\
\hline & $5 f$ & $-0,020011$ & & $5 f$ & $-0,020009$ & 0,0 \\
\hline & $6 f$ & $-0,013895$ & & $6 f$ & $-0,013895$ & 0,0 \\
\hline & $7 f$ & $-0,010208$ & & $7 f$ & $-0,010208$ & 0,0 \\
\hline & $8 f$ & $-0,0078171$ & & $8 f$ & $-0,0078155$ & 0,0 \\
\hline
\end{tabular}

par uné valeur moyenne, de telle sorte que l'on puisse calculer $\left\langle\beta, m\left|T U_{\text {eff }} T^{+}\right| \alpha, m\right\rangle$ en employant un potentiel moyen central $\bar{U}_{\text {eff }}$ que nous prendrons de la forme :

$$
\bar{U}_{\mathrm{eff}}=-\frac{z}{r}-\frac{1}{r}[\bar{Z}-z+\alpha r] \mathrm{e}^{-\bar{\sigma} r}
$$

(Nous prenons une expression contenant un terme supplémentaire pour avoir suffisamment de souplesse dans la détermination des paramètres).

Bien entendu cela constituera encore une approximation, mais qui sera de même nature pour tous les vecteurs $|\alpha, m\rangle$ de la base, contrairement à la troncature sur les valeurs de $\ell$. Par ailleurs, comme l'opérateur $H_{1}$ représente uniquement la polarisation de $\mathrm{M}^{(z-1)+}$ (c'està-dire que l'électron ne peut pas se trouver sur l'autre centre), il n'est pas physiquement absurde de représenter l'influence de $\mathrm{M}^{z+}$ sous la forme d'un potentiel central (du moins pour des valeurs de $R$ suffisamment grandes qui correspondent au domaine de validité de notre modèle). 
En effet, la non-localité décrit essentiellement les effets d'échange entre l'électron actif et le cœur $\mathbf{M}^{z+}$. Or ici l'électron actif ne se trouve pas sur le centre $\mathbf{M}^{z+}$ mais sur l'ion $\mathbf{M}^{(z-1)+}$; donc les effets d'échange avec $\mathbf{M}^{z+}$ sont négligeables. Par conséquent, seule la partie centrale du potentiel sera significative.

Il faut alors se donner une méthode pour « extraire » en quelque sorte la partie centrale $\bar{U}_{\text {eff }}$ de l'opérateur non local $U_{\text {eff }}$ Par ailleurs, $\bar{U}_{\text {eff }}$ doit représenter le potentiel central qui ressemble le plus à $U_{\text {eff }}$ mais relativement à la base atomique $|n \ell m\rangle$.

Ceci conduit à introduire une sorte d'écart quadratique défini par :

$$
\Delta(\bar{Z}, \alpha, \bar{\sigma})=\sum_{n, \ell}\left\langle n, \ell\left|\left[\bar{U}_{\text {eff }}(\bar{Z}, \alpha, \bar{\sigma})-U_{\ell}\right]^{2}\right| n, \ell\right\rangle .
$$

En minimisant la fonction $\Delta$ par rapport aux trois paramètres $\bar{Z}$, $\alpha$ et $\bar{\sigma}$, on obtiendra la partie centrale du potentiel initial $U_{\text {eff }}$.

On montre que ce calcul se ramène à trouver le zéro d'une seule fonction en $\bar{\sigma}$, car la minimisation sur $\bar{Z}$ et $\alpha$ peut être résolue analytiquement.

On vérifie alors que le potentiel central ainsi obtenu est une bonne approximation, en recalculant le spectre de l'hamiltonien atomique avec ce nouveau potentiel central. La vérification sur les cas étudiés montre qu'on obtient un spectre moins proche de l'expérience, ce qui est normal, mais qui ne s'écarte pas à plus de $10 \%$ des valeurs expérimentales, l'origine des énergies étant l'énergie d'ionisation. Ce pourcentage représente la contribution non locale au potentiel global. Dans la suite nous vérifions, dans le cas du sodium, pour lequel on connaît les courbes moléculaires, que cette approximation donne des résultats satisfaisants.

\section{Applications aux ions $\mathrm{Na}_{2}^{+}$et $\mathrm{Ba}_{2}^{3+}$.}

Dans un premier temps nous avons appliqué le formalisme calculatoire à l'ion $\mathrm{Na}_{2}^{+}$que l'on connaît bien, tant au point de vue expérimental qu'au point de vue théorique. La comparaison entre nos résultats et ces données constitue un test pour le formalisme que nous avons présenté.

Dans un deuxième temps, nous reportons des résultats sur les termes de potentiel de l'ion $\mathrm{Ba}_{2}^{3}{ }^{+}$. Le formalisme «non local avec état de cœur », que nous présentons dans cet article, a été entrepris car les études que nous avions faites précédemment [9] sur les ions hétéronucléaires tels que $\mathrm{NaBa}^{2+}, \mathrm{HBa}^{2+}, \ldots$, où un potentiel local avec état de cœur avait été proposé, ont montré les limites du domaine d'application d'un formalisme local avec état de cœur.

En effet, le modèle non local [9] a donné de bons résultats pour les ions hétéronucléaires car il suffisait de décrire parfaitement un des cœurs, en l'occurrence celui de l'alcalin, la description du deuxième cœur celui de l'alcalino-terreux ayant une importance du deuxième ordre. Par contre, dans le cas d'un système homonucléaire où deux cœurs de baryum entrent en jeu, il s'est avéré nécessaire de prendre un modèle plus performant pour décrire ce système.

1. L'ION $\mathrm{Na}_{2}^{+}$. - i) Dans une première phase nous avons appliqué le formalisme aux calculs d'états moléculaires $\Sigma$ de l'ion $\mathrm{Na}_{2}^{+}$en utilisant un pseudo-potentiel local du type Hellmann dont les paramètres sont indiqués dans le tableau Ia. On peut remarquer que l'on trouve une valeur négative de $Z$ dans le potentiel atomique, ce qui traduit le comportement répulsif du potentiel aux faibles valeurs de $r$. Par ailleurs, ces résultats montrent à l'évidence que les erreurs qu'apporte la contrainte que l'on choisit de s'imposer, à savoir prendre un potentiel 
central, ne sont pas très importantes sur l'ensemble du spectre. On peut néanmoins remarquer la détérioration des résultats sur la série $\ell=0$.

Les résultats relatifs à l'état fondamental $\Sigma_{\mathrm{g}}(3 \mathrm{~s})$ de l'ion $\mathrm{Na}_{2}^{+}$sont représentés sur la figure 2. La profondeur du puits $D_{\mathrm{e}}$ et la distance d'équilibre $R_{\mathrm{e}}$ sont respectivement - 0,2292 u.a. (ou 1,01 eV) et 6,5 u.a. (ou 0,36 nm). Ces valeurs approchent les valeurs expérimentales [13-14] (0,985 eV et $0,36 \mathrm{~nm})$, l'écart étant de 2,5\% pour l'énergie ; dans le tableau II sont aussi reportées les différentes valeurs expérimentales et théoriques publiées dans la littérature.

En conclusion, on remarque que l'emploi de ce pseudo-potentiel représente une légère amélioration par rapport aux résultats de la référence [5] qui utilisait le même type de potentiel mais obtenu en s'imposant d'autres contraintes. Cependant, conformément aux résultats généraux sur les potentiels atomiques, on vérifie que la modélisation via un pseudopotentiel est rigide car elle fait supporter tous les effets au potentiel. Néanmoins, ces résultats moléculaires présentent une précision du même ordre de grandeur que celle du modèle atomique de départ.

ii) Dans une deuxième phase, un potentiel non local avec états de cœur est utilisé dans le modèle moléculaire. La représentation de l'atome est décrite par un potentiel non local possédant deux états de cœur $1 \mathrm{~s}$ et $2 \mathrm{p}$. La correspondance entre les premiers états du modèle et ceux de l'atome est consignée dans le tableau Ib.

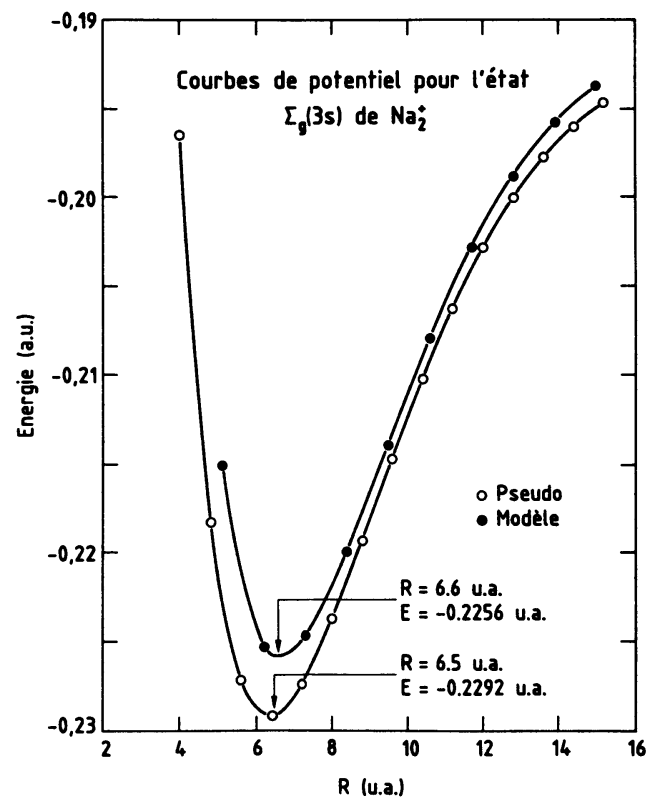

Fig. 2. - Courbes de potentiel de l'état fondamental $\Sigma_{\mathrm{g}}(3 s)$ de l'ion moléculaire $\mathrm{Na}_{2}^{+}$pour deux représentations différentes. Les points pleins $(\bullet)$ représentent les résultats obtenus en utilisant le potentiel-modèle, alors que les points blancs $(0)$ représentent les résultats correspondant au pseudopotentiel, l'écart entre les valeurs des puits est inférieur au $1 / 100 \mathrm{eV}$.

$\left[\Sigma_{g}(3 s)\right.$ ground state potential curves for $\mathrm{Na}_{2}^{+}$ion. The calculated values correspond to the Model Potential $(\bullet)$ and the pseudo-potential $(O)$. The difference between the well depth values is less than $1 / 100 \mathrm{eV}$. 
Les données relatives à ce potentiel sont indiquées dans le tableau $\mathrm{Ib}$, on remarque qu'il a été possible de diminuer le nombre de paramètres du potentiel en imposant à tous les $\sigma_{\ell}$ d'avoir la même valeur $\sigma$, de telle façon que $1 / \sigma$ puisse représenter la portée isotrope des effets de cœur. En outre, on constate un bon accord entre le modèle et l'expérience, pour un nombre de paramètres mis en jeu relativement faible. Par ailleurs, on peut remarquer le faible caractère non local du potentiel, puisque à l'exception des états $\mathrm{p}$, on trouve la même valeur de $Z_{\ell}$ voisine de $Z_{\ell}=2,5$.

Les calculs relatifs à ce choix de potentiel-modèle non local à deux états de cœur, donnent pour l'état fondamental (non virtuel) noté $\Sigma_{\mathrm{g}}(2 \mathrm{~s})$ pour le modèle et correspondant à $\Sigma_{\mathrm{g}}(3 \mathrm{~s})$ pour l'ion moléculaire réel, les résultats suivants : $R_{\mathrm{e}}=6,6 \mathrm{u} . \mathrm{a} .(0,35 \mathrm{~nm})$ et $D_{\mathrm{c}}=0,2256$ u.a. $(1,001 \mathrm{eV})$. Ces données sont reportées sur le tableau II ; en comparant ces derniers résultats aux résultats expérimentaux, l'erreur est de $3 \%$ sur $R_{\mathrm{e}}$ et $1,5 \%$ sur $D_{\mathrm{c}}$. Nous avons dans ces calculs omis le terme à trois corps, or nous savons, d'après l'étude de Henriet et Masnou, que la tendance en incluant ces termes, est de réduire la profondeur du puits de $4 \%$ et de pousser vers des plus grandes valeurs la position d'équilibre $(1,5 \%)$ pour $R$.

Utilisant ces corrections on trouve : $R_{\mathrm{e}}=0,355\left(\Delta R_{\mathrm{e}}=1,5 \%\right)$ et $D_{\mathrm{e}}=0,86\left(\Delta D_{\mathrm{e}}=2 \%\right)$, les $\Delta$ représentent les écarts par rapport aux résultats expérimentaux $[13,14]$.

Dans tous les calculs du terme à trois corps, il existe une difficulté sur la détermination des paramètres de coupure qui sont introduits dans les termes de polarisation pour éviter les divergences à l'origine, ce problème a été traité dans les références $[4,6,7]$ et partiellement résolu en faisant appel à des grandeurs calculées $a b$ initio dans le cas du néon [8-12]. Cependant, à l'heure actuelle, ce problème reste très délicat lorsque l'on demande des précisions inférieures au $1 \%$ et n'a pas trouvé de solution qui ne souffre d'aucune ambiguïté.

C'est une des raisons qui nous a fait, dans l'étude de ce formalisme, négliger le terme à trois corps car il représente en lui-même une étude à part.

D'autre part, la description atomique supportant au départ une imprécision de l'ordre de $1 \%$ sur les cinq premiers états $p$, il ne semble pas judicieux d'introduire dans l'hamiltonien un terme à trois corps dont l'apport corrige les erreurs d'un ordre de grandeur de $3 \%$.

Tableau II. - Profondeur et position du puits de potentiel de l'ion $\mathrm{Na}_{2}^{+}$.

[Well depths and positions of $\mathrm{Na}_{2}^{+}$molecular ion.]

\begin{tabular}{|c|c|c|c|c|}
\hline $\begin{array}{c}\text { Références } \\
\text { Théorie }\end{array}$ & & $D_{\mathrm{e}}(\mathrm{eV})$ & $R_{\mathrm{e}}(\mathrm{nm})$ & $\begin{array}{c}\text { Terme à } \\
\text { trois corps }\end{array}$ \\
\hline [1] & & 1,02 & 0,354 & non \\
\hline [5] & & 1,02 & 0,328 & non \\
\hline [15] & & 1,02 & 0,356 & non \\
\hline [6] & & 1,02 & 0,355 & non \\
\hline [15] & & 0,993 & 0,359 & oui \\
\hline [16] & & 0,98 & 0,33 & oui \\
\hline \multirow{2}{*}{ [6] } & & 0,980 & 0,360 & oui \\
\hline & modèle & 1,001 & 0,350 & non \\
\hline \multicolumn{5}{|l|}{ Notre calcul } \\
\hline & pseudo & 1,01 & 0,355 & non \\
\hline \multicolumn{5}{|l|}{ Expérience } \\
\hline [13] & & 0,984 & 0,36 & \\
\hline$[14]$ & & 0,986 & 0,36 & \\
\hline
\end{tabular}


On se borne donc dans cet article à développer et à tester un formalisme contenant conjointement une « $\ell$ » dépendance, des états virtuels de cœurs, et une base hydrogénoïde, ce qui n'a fait l'objet d'aucune publication à l'heure actuelle. Enfin, le but final étant de calculer les ions moléculaires du baryum, les données ab initio, ou semi-empiriques pour le comportement des termes de polarisation à l'origine étant mal connues, il est prématuré dans un premier temps de prendre en compte dans le formalisme ces contributions qui représentent en fait un raffinement que nous ne pourrons complètement gérer.

2. L'ION $\mathrm{Ba}_{2}^{3+}$. - En ce qui concerne le baryum, nous avons utilisé le même type de potentiel non local avec trois états de cœur (1s, $2 \mathrm{~s}$ et $2 \mathrm{p})$ décrivant l'interaction d'un électron avec $\mathrm{Ba}^{2+}$. Les caractéristiques de ce potentiel sont publiées en [10] et résumées dans la table III.

L'utilisation de ce potentiel pour le calcul des états diexcités de l'atome de baryum a montré la fiabilité de cette description [10]. On remarque en observant les résultats relatifs à l'interaction $\mathrm{e}-\mathrm{Ba}^{2}+$ (Tab. III), que la base hydrogénoïde utilisée comporte 6 orbitales de type s, 5 de type p, 4 de type $d$ et 4 de type $\mathrm{f}$; ce qui équivaut en termes d'orbitales de Slater à 25 de type $\mathrm{s}, 15$ de type $\mathrm{p}, 10$ de type d et 10 de type $\mathrm{f}$. Les problèmes de convergence relatifs à la valeur du palier de l'état fondamental et à son extension spatiale, ont été résolus avec cette base d'une part ; d'autre part les orbitales $p$ et $d$ de la base assurent la prise en compte des effets de polarisation dipolaire et quadripolaire.

Tableau III. - Mêmes commentaires que les tableaux Ia et Ib pour le potentiel-modèle du $\mathrm{Ba}^{+}$.

[This table corresponds to the Helmann model-potential of $\mathrm{Ba}^{+}$ion.]

\begin{tabular}{|c|c|c|c|c|c|c|}
\hline & $\mathrm{Ba}^{+}$ & $E^{\exp }$ (u. a. ) & & $h$ & $E^{\mathrm{c}}$ (u. a. $)$ & $\%$ erreu \\
\hline$Z_{0}=3,471$ & & & Cœur & 1s & $-5,4828$ & - \\
\hline$\sigma_{0}=0,4$ & & & Cœur & $2 s$ & $-1,0785$ & 一 \\
\hline & $6 s$ & $-0,3676$ & & $3 s$ & $-0,3676$ & 0,0 \\
\hline & $7 \mathrm{~s}$ & $-0,1746$ & & $4 s$ & $-0,1741$ & 0,3 \\
\hline & $8 \mathrm{~s}$ & $-0,1032$ & & $5 s$ & $-0,1022$ & 0,9 \\
\hline & $9 \mathrm{~s}$ & $-0,06836$ & & $6 s$ & $-0,06736$ & 1,5 \\
\hline \multicolumn{7}{|l|}{$Z_{1}=2,655$} \\
\hline$\sigma_{1}=0,4$ & & & Cœur & $2 p$ & $-0,6955$ & - \\
\hline & $6 \mathrm{p}$ & $-0,2702$ & & $3 p$ & $-0,2702$ & 0,0 \\
\hline & $7 p$ & $-0,1407$ & & $4 p$ & $-0,1421$ & 1,0 \\
\hline & $8 p$ & $-0,08726$ & & $5 p$ & $-0,08809$ & 0,9 \\
\hline & $9 p$ & - & & $6 p$ & $-0,06005$ & 一 \\
\hline \multicolumn{7}{|l|}{$\begin{array}{l}Z_{2}=3,579 \\
\sigma_{2}=0,4\end{array}$} \\
\hline & $5 d$ & $-0,3432$ & & $3 d$ & $-0,3432$ & 0,0 \\
\hline & $6 d$ & $-0,1577$ & & $4 d$ & $-0,1602$ & 1,5 \\
\hline & $7 d$ & $-0,09491$ & & $5 d$ & $-0,09525$ & 0,3 \\
\hline & $8 d$ & $-0,06370$ & & $6 d$ & $-0,06356$ & 0,2 \\
\hline \multirow{2}{*}{\multicolumn{7}{|c|}{$\begin{array}{l}Z_{3}=14,31 \\
\sigma_{3}=0,94\end{array}$}} \\
\hline & & & & & & \\
\hline & $4 f$ & $-0,1472$ & & $4 f$ & $-0,1472$ & 0,0 \\
\hline & $5 f$ & $-0,1055$ & & $5 \mathrm{f}$ & $-0,1136$ & 7,1 \\
\hline & $6 f$ & $-0,07305$ & & $6 f$ & $-0,07260$ & 0,6 \\
\hline & $7 f$ & $-0,05216$ & & $7 f$ & $-0,05048$ & 3,3 \\
\hline
\end{tabular}


Les résultats du calcul moléculaire des états $\Sigma$ de l'ion $\mathrm{Ba}_{2}^{3+}$ sont représentés sur la figure 3 en ce qui concerne l'état fondamental $\Sigma_{\mathrm{g}}(6 \mathrm{~s})$ et repris dans la figure 4 avec les états excités. Ils ont été obtenus en diagonalisant l'hamiltonien dans une base de 19 états hydrogénoïdes. Les éléments de matrice sont exprimés analytiquement en utilisant les coordonnées sphéroïdales. Les calculs ont été réalisés sur un micro-ordinateur en une dizaine de minutes, en langage Pascal.

Cet ion moléculaire est caractérisé en première approximation par la répulsion coulombienne entre deux ions de charges +2 chacun, qui est énergétiquement plus forte que tout effet chimique de liaison. Cependant, les forces associées aux effets de liaisons peuvent être du même ordre de grandeur que les forces répulsives de Coulomb, ainsi on peut noter que l'état fondamental $\Sigma_{\mathrm{g}}(6 \mathrm{~s})$ ne possède pas de véritable puits de potentiel, il apparaît seulement un palier entre 10 et 13 u.a.

En ce qui concerne les états excités $\Sigma_{\mathrm{g}, \mathrm{u}}(5 \mathrm{~d})$ et $\Sigma_{\mathrm{g}, \mathrm{u}}(6 \mathrm{p})$, on ne voit pas apparaître cet effet, la répulsion en $+4 / R$ étant dominante.

\section{Conclusion.}

Dans le cadre de la méthode de combinaisons linéaires d'orbitales atomiques, nous avons développé un calcul formel qui permet de séparer analytiquement les différentes contributions énergétiques du processus de liaison, à sạvoir l'énergie atomique, l'énergie de polarisation et l'énergie de mise en commun de la charge. Dans chaque sous-espace de moment magnétique et de parité donné, l'hamiltonien moléculaire $\mathrm{H}$ peut donc s'écrire sous la forme :

$$
H=H_{1}+\varepsilon H_{2} \quad(\varepsilon= \pm 1),
$$

$H_{1}$ décrivant la polarisation de $\mathrm{M}^{(z-1)+}$ par $\mathrm{M}^{z+}$ et $H_{2}$ la mise en commun de l'électron entre les deux centres. Nous avons ainsi vérifié le comportement aux grandes distances internucléai-

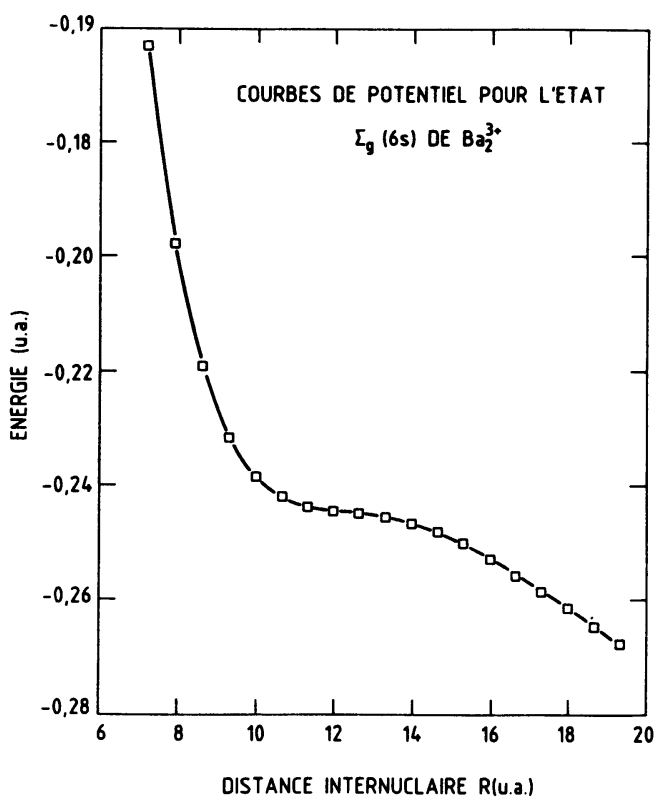

Fig. 3. - Etat fondamental de l'ion $\mathrm{Ba}_{2}^{3+}$.

[Ground state potential curve of $\mathrm{Ba}_{2}^{3+}$ ion.] 


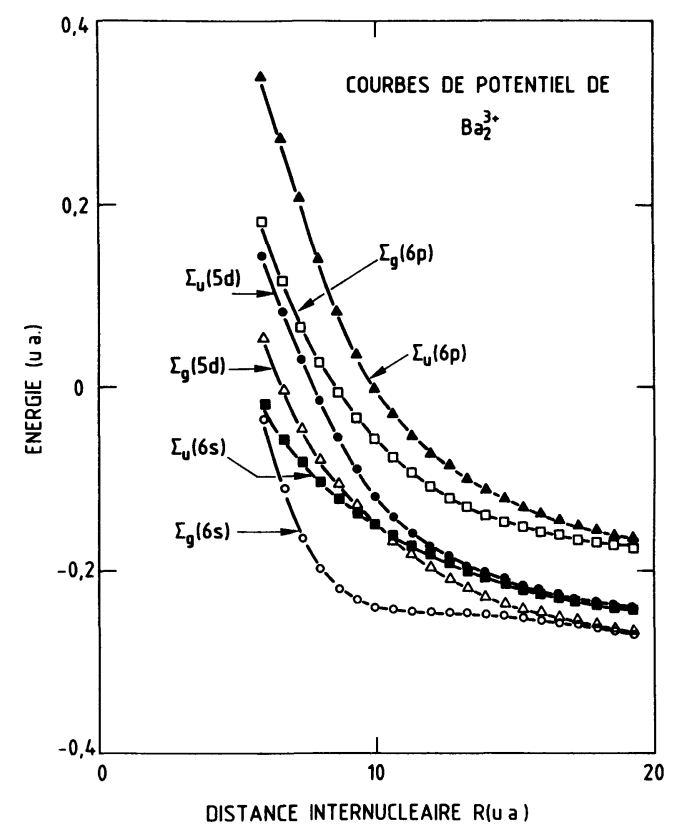

Fig. 4. - Etat fondamental et premiers états excités de l'ion $\mathrm{Ba}_{2}^{3+}$.

[Ground state and low lying states of $\mathrm{Ba}_{2}^{3+}$ ion.]

res de $H_{1}$ et $H_{2}$ (coulombien et exponentiel). Par ailleurs, on remarque que les calculs ne font intervenir que trois types d'éléments de matrice relatifs aux opérateurs $\mathrm{T}, U_{\text {eff }} T$ et $T U_{\text {eff }} T^{+}$.

Grâce à ce formalisme, il est possible de comprendre le rôle physique des éléments de matrice des opérateurs $U_{\text {eff }} T$ et $T U_{\text {eff }} T^{+}$. On peut alors traiter correctement l'effet de troncature de la série en $\ell$ dans $U_{\text {eff }}$, en remarquant que ceci est spécifique de l'hamiltonien de polarisation $H_{1}$ et n'intervient nullement dans l'opérateur $H_{2}$.

Nous avons appliqué cette méthode de calcul à un système connu : l'ion moléculaire $\mathrm{Na}_{2}^{+}$, pour deux représentations atomiques différentes. Nous avons montré que les résultats obtenus étaient en conformité, avec les résultats théoriques et expérimentaux connus. Enfin, nous donnons les courbes de potentiel $\Sigma$ prévues pour l'ion moléculaire $\mathrm{Ba}_{2}^{3+}$.

L'ensemble de ces résultats relatifs à $\mathrm{Ba}_{2}^{3}{ }^{+}$nous permet de prédire que le système à deux électrons actifs, tel que $\mathrm{Ba}_{2}^{2}{ }^{+}$, présenterait un état pseudo-lié, à grand temps de vie, accroché sur la décroissance coulombienne en $+2 / R$ du système. Cela avait été observé pour certains états des ions deux fois chargés $\mathrm{BaX}^{2+}$ (où $X=H, \mathrm{Na}, \mathrm{K}, \mathrm{Rb}, \mathrm{Cs} . .$. ) [9]. Le système $\mathrm{Ba}_{2}^{2}+$ est en cours d'étude car il présente la propriété intéressante de posséder pour troisième état moléculaire, situé après le fondamental de type $6 \mathrm{~s}^{2}$ et le premier excité de type $6 \mathrm{~s} 5 \mathrm{~d}$, un diexcité de type $5 \mathrm{~d}^{2}$. Cette observation est tout à fait spécifique à certains alcalinoterreux, alors qu'en général l'état diexcité se trouve à un niveau très éloigné du fondamental, comme cela est le cas pour le premier état diexcité de sodium $\mathrm{Na}_{2}(3 p, 3 p)$, qui se trouve être le sixième état d'excitation. 


\section{Bibliographie}

[1] Bardsley J. N., Case Stud. At. Phys. 4 (1974) 299.

[2] Dixon R. N. and Robertson I. L., Spect. Period. Rep. Theor. Chem. 3 (1978) 100.

[3] Peach G., Com. At. Mol. Phys. D XI (1982) 117.

[4] Bottcher C. and Dalgarno A., Proc. R. Soc. London A 340 (1974) 187.

[5] Valance A., Phys. Lett., 59A (1976) 271 ; J. Chem. Phys. 69 (1978) 355.

[6] Henriet A. and Masnou-Seeuws F., Chem. Phys. Lett. 101 (1983) 535.

[7] Philippe M. Masnou-Seeuws F. and Valiron P., J. Phys. B 12 (1979) 2493.

[8] Valance A., Rahal H., Runge S. and Murez C., J. Phys. B 19 (1986) 3919.

[9] Valance A., Bernier A. and Bergeron H., J. Phys. B 19 (1986) 857.

[10] Bergeron A. and Valance A., Z. Phys. D. At. Mol. Clusters 6 (1987) 309.

[11] Bergeron H., Thèse de Docteur ès Sciences, $n=465$, Orsay (1988).

[12] Valance A., Rahal H. and Murez C., Chem. Phys. 97 (1985) 63.

[13] Carlson N. W., Taylor A. J., Schalow A. L., Phys. Rev. Lett. 45 (1980) 18.

[14] Martin S., Chevaleyre J., Valignat S., Perror J. P., Broyer M., Cabaud M., et Hoareau A., Chem. Phys. Lett. 87 (1982) 235.

[15] Fuentealba P., Preuss H., Stoll H. and Von Szentpaly L., Chem. Phys. Lett. 89 (1982) 418.

[16] Cerjan C. J., Docken K. K., Dalgarno A., Chem. Phys. Lett. 38 (1976) 401. 